\title{
Population Pharmacokinetics of Tadalafil in Pediatric Patients with Pulmonary Arterial Hypertension: A Combined Adult/Pediatric Model
}

\author{
Lisa Ferguson-Sells ${ }^{1}\left[\right.$ D Nieves Velez de Mendizabal ${ }^{1} \cdot$ Baohui $i^{1} \cdot$ David Small $^{1}$
}

Accepted: 11 June 2021 / Published online: 11 August 2021

(c) The Author(s) 2021

\begin{abstract}
Background Tadalafil $40 \mathrm{mg}$ once daily is approved for adult patients with pulmonary arterial hypertension (PAH). To investigate and potentially fulfill an unmet need in pediatric patients with $\mathrm{PAH}$, pharmacokinetic (PK) data were explored in a pediatric phase Ib/II study and pooled with prior phase III (pulmonary arterial hypertension and response to tadalafil [PHIRST-1]) adult data to develop the first population PK model for tadalafil in pediatric patients with PAH.

Methods H6D-MC-LVIG (NCT01484431) was an open-label, multicenter, multiple ascending dose study in pediatric patients with PAH, while PHIRST-1 was a phase III, multicenter, randomized, double-blind, placebo-controlled, parallel design study in adults with PAH who received one of five treatments (tadalafil 2.5, 10, 20, or $40 \mathrm{mg}$, or placebo orally, once daily). PK data from the studies were pooled to develop a pediatric population PK model for tadalafil that characterized relationships among dose, exposure, and the effects of covariates with an aim to develop a population PK model that could simulate concentration-time profiles and assess exposure-matched dosing strategies in a pediatric PAH population.

Results In line with the observed data, modeling and simulation demonstrated that the doses studied in the pediatric population produced area under the concentration-time curves (AUCs) within the range of those associated with improved exercise ability in adults with PAH. The analyses included 1430 observations from 305 adult patients (PHIRST-1: 69 males and 236 females, 1102 observations) and 19 pediatric patients (LVIG: 6 males and 13 females, 328 observations) who received tadalafil once daily at different dose levels. The best-fit base model retained an effect of weight on apparent volume of distribution $(V / F)$, fixed to the allometric scaling value of 1 , and did not include an effect of weight on apparent clearance $(C L / F)$. Other covariate effects were that bosentan increased $C L / F, V / F$ decreased with decreasing body weight, and bioavailability $(F)$ decreased with increasing dose and decreasing age. The PK model reliably predicted the observed concentrations and overall variability evident from the overlap of the individual observed concentrations with the distributions of simulated concentrations.

Conclusions A one-compartment model parameterized in terms of $F$, absorption rate constant, $C L / F$, and $V / F$ described the data well. The model demonstrated that plasma tadalafil concentrations in pediatric patients aged 2 to $<18$ years were similar to those in adults at similar doses, and confirmed that dosing of $40 \mathrm{mg}$ once daily in pediatric patients with a bodyweight $\geq 40 \mathrm{~kg}$, and a dose of $20 \mathrm{mg}$ once daily in patients with a body weight $<40 \mathrm{~kg}$ and aged $\geq 2$ years are suitable for phase III evaluation.
\end{abstract}

Trial Registration Number (Date of Registration) LVIG: ClinicalTrials.gov identifier: NCT01484431 (2 December 2011). PHIRST-1: ClinicalTrials.gov identifier: NCT00125918 (2 August 2005).

Extended author information available on the last page of the article 


\section{Key Points}

In a phase Ib/II study of tadalafil in pediatric patients aged $\geq 2$ years with pulmonary arterial hypertension (PAH), population pharmacokinetic modeling and simulation showed that a dose of $40 \mathrm{mg}$ once daily in pediatric $\mathrm{PAH}$ patients with a body weight $\geq 40 \mathrm{~kg}$ and a dose of $20 \mathrm{mg}$ once daily in pediatric PAH patients with a body weight $<40 \mathrm{~kg}$ produced tadalafil plasma concentrations within the range of those associated with improved exercise ability in adult PAH patients.

These doses are recommended for further investigation in pediatric PAH patients who are at least 2 years of age.

Plasma tadalafil concentrations observed in pediatric patients aged 2 to $<18$ years were similar to adult concentrations at similar doses.

The population pharmacokinetics of tadalafil in pediatric patients aged 2 to $<18$ years were described by a linear one-compartment model.

\section{Introduction}

Pulmonary arterial hypertension (PAH) is a chronic and progressive disease characterized by the elevation of pulmonary arterial pressure and pulmonary vascular resistance, leading to right heart failure and death $[1,2]$. Despite the treatment progress in adults, approved therapies for $\mathrm{PAH}$ in children are lacking. The therapies approved for adults are often used off-label in pediatric patients [3] and therefore it is important to evaluate the safety and drug exposure of such treatments in this younger population.

Tadalafil is a potent and selective phosphodiesterase 5 inhibitor approved at an oral dose of $40 \mathrm{mg}$ once daily to improve exercise ability in adult patients with PAH. Tadalafil improved clinical outcomes and quality of life and delayed the time to clinical worsening in an adult PAH patient trial (pulmonary arterial hypertension and response to tadalafil [PHIRST-1], also referred to as H6D-MC-LVGY [LVGY]) [4]. Due to the rarity of the disease, large trials for investigational PAH treatments in pediatric patients are not feasible; therefore it is important to leverage the available data with modeling and simulation to support current investigation.

A phase Ib/II, multiple ascending dose study (H6D-MCLVIG [LVIG]) [5] was conducted to evaluate the safety and pharmacokinetics $(\mathrm{PK})$ of tadalafil in planned $\mathrm{PAH}$ patients aged 6 months to $<18$ years, with a goal to mimic typical drug exposures in adults with PAH and determine doses for further investigation in phase III. A previous publication discussed safety and noncompartmental analyses for this trial [5], whereas the focus of this article is on the results of the population PK (PopPK) analysis. The purpose of the PopPK analysis was to characterize tadalafil PK across the range of body weights and ages enrolled in the study and in each cohort; to evaluate the effect of various covariates such as age, body weight, sex, and endothelin receptor antagonist (ERA) use on tadalafil exposure; and to predict appropriate dose(s) in subsequent pediatric studies of tadalafil. LVIG collected PK samples from 19 pediatric patients with PAH. The primary PK objectives of LVIG were to characterize the $\mathrm{PK}$ of tadalafil in the pediatric $\mathrm{PAH}$ population, evaluate the effects of clinically relevant covariates, compare tadalafil PK in pediatric PAH patients with the historical PK data in adult patients with $\mathrm{PAH}$, and to inform tadalafil dosing in this pediatric population. An exposure-match approach based on allometric scaling $[6,7]$ was used to predict tadalafil doses for the pediatric population before starting the study; then, during the study, exposures were assessed in each patient at each dose using noncompartmental analyses [5]. The target exposure in pediatric patients was the 24-h area under the curve (AUC) estimated by PopPK analyses of sparsely sampled data in adult patients with PAH (PHIRST-1; the adult model) [4]. This target AUC was $14825 \mathrm{ng} \cdot \mathrm{h} / \mathrm{mL}$ in adult patients with PAH taking the approved dose of $40 \mathrm{mg}$ tadalafil in PHIRST-1.

The pediatric PK data from LVIG were assessed using modeling and simulation to select doses for a larger phase III clinical trial in pediatric patients with PAH. Due to a paucity of tadalafil PK data in pediatric patients with $\mathrm{PAH}$ in the LVIG study, the PK data from LVIG were combined with the larger PK dataset from the PHIRST-1 study in adult patients with PAH. The pooled dataset was used to develop a pediatric PopPK model (the pediatric model) for tadalafil that characterized relationships among dose, exposure, and the effects of covariates, with an aim to develop a model that can be used to simulate concentration-time profiles and investigate exposurematched dosing strategies in a pediatric $\mathrm{PAH}$ population.

The focus of this article is the PK of tadalafil in pediatric patients with PAH, which was evaluated using the first PopPK model developed for tadalafil in this population.

\section{Methods}

\subsection{Relevant Pharmacokinetic Information for Tadalafil}

The maximum plasma concentration $\left(C_{\max }\right)$ of tadalafil occurs $2-8 \mathrm{~h}$ (median $4 \mathrm{~h}$ ) after a single oral dose; however, the absolute bioavailability (F) of tadalafil has not been determined. The rate and extent of tadalafil absorption are not influenced by food. The mean $V / F$ following an oral dose is $77 \mathrm{~L}$, indicating that tadalafil distributes into tissues. At therapeutic concentrations, 
94\% of tadalafil in plasma is bound to proteins. Tadalafil is predominantly metabolized by cytochrome $\mathrm{P} 450$ (CYP) $3 \mathrm{~A}$ to a catechol metabolite. In vitro data suggest that metabolites are not pharmacologically active at clinical concentrations. Data suggest that patients with pulmonary hypertension have lower clearance of tadalafil compared with healthy volunteers. Following a 40-mg dose in healthy subjects not taking bosentan, the mean $C L / F$ of tadalafil is $3.4 \mathrm{~L} / \mathrm{h}$ and the mean terminal halflife is $15 \mathrm{~h}$. In adult patients with pulmonary hypertension not receiving concomitant bosentan, the mean $C L / F$ for tadalafil is $1.6 \mathrm{~L} / \mathrm{h}$ and the mean terminal half-life is $35 \mathrm{~h}$ [8]. In healthy males, the $C L / F$ of tadalafil increased by about $70 \%$ with concomitant bosentan [9]. Tadalafil is excreted predominantly as metabolites, mainly in the feces ( $61 \%$ of the dose) and, to a lesser extent, in the urine (36\% of the dose) [8].

A relative bioavailability study was conducted in healthy adults to compare tadalafil tablets and an experimental oral suspension. Maximum concentrations of tadalafil were $23 \%$ lower $(90 \%$ confidence interval [CI] of the ratio: 0.716-0.829) and occurred $1 \mathrm{~h}$ later for the 20-mg suspension formulation compared with the 20-mg tablet, but tadalafil AUC from time zero to infinity $\left(\mathrm{AUC}_{\infty}\right)$ did not differ. The suspension produced a less-than-dose-proportional increase in tadalafil exposure over the 20 - to $40-\mathrm{mg}$ dose range, which is also seen with the tablet formulation. This disproportionality was comparable in bosentan users and those receiving tadalafil alone.

In adult PAH patients, subjects who benefited most from tadalafil treatment received doses of at least $20 \mathrm{mg}$ once-daily, irrespective of the PK influence of bosentan and other disease- or subject-specific factors.

\subsubsection{Tadalafil Bioanalytical Methods}

Human plasma samples were analyzed for tadalafil using a validated atmospheric pressure chemical ionization (APCI) liquid chromatographic method with tandem mass spectrometry (LC-MS/MS) with a lower limit of quantification (LLOQ) of $0.500 \mathrm{ng} / \mathrm{mL}$. The adult assay required a $200 \mu \mathrm{L}$ plasma sample, and the assay was revalidated for pediatric trials to accommodate a smaller $50 \mu \mathrm{L}$ plasma sample. All samples were analyzed at the same bioanalytical laboratory. Blood samples in both pediatric and adult participants were collected intravenously.

\subsection{Studies Included in the Analysis}

Two studies (LVIG and PHIRST-1) were included in the pooled adult/pediatric population analysis:
- LVIG (ClinicalTrials.gov identifier: NCT01484431) was a phase Ib/II, open-label, multicenter, international, multiple ascending dose study in pediatric patients with PAH [5]. The study evaluated the PK of tadalafil administered orally once daily, as a tablet or suspension, with two doses evaluated in each patient. LVIG was conducted in two parts: Periods 1 and 2. In Period 1, 19 pediatric patients were evaluated for PK and safety/tolerability of tadalafil during the first 10 weeks of treatment. Patients continued into Period 2 for at least 2 years for safety evaluation. One PK sample was collected within 3 months after the start of Period 2; 15 patients had these data available at the time of analysis and were subsequently included in this analysis. Further information for Period 2 is beyond the scope of this manuscript. All patients were categorized into three cohorts based on body weight: heavy-weight ( $\geq 40 \mathrm{~kg}$ ), middle-weight ( 25 to $<40 \mathrm{~kg}$ ), and light-weight $(<25 \mathrm{~kg})$. Each patient received tadalafil once daily for 10 weeks in Period 1: 5 weeks at a low dose and then 5 weeks at a high dose [5]. The low or high doses selected for each cohort were intended to produce tadalafil exposures comparable with those produced by $5-10 \mathrm{mg}$ once daily or $20-40 \mathrm{mg}$ once daily, respectively, in adult patients. Children in the middleand heavy-weight groups received commercially available tablets, whereas children in the light-weight group received an oral suspension. No patient under 2 years of age was enrolled [5].

- PHIRST-1 (ClinicalTrials.gov identifier: NCT00125918) was a phase III, 16-week, multicenter, randomized, double-blind, placebo-controlled, parallel-design study in patients aged $>12$ years with PAH [4]. The primary efficacy outcome measure was placebo-corrected change from baseline to week 16 in 6-min walk distance. Adult patients $(n=406)$ were randomly assigned to receive one of five treatments (tadalafil 2.5, 10,20, or $40 \mathrm{mg}$, or placebo, once daily). Since this trial enrolled just one patient below 18 years of age (age 14 years), this population will be referred to as adult for the purposes of clarity between the two studies. A secondary objective of the study was to evaluate tadalafil PopPK; thus, sparse PK samples were collected and used to develop a PopPK model that characterized the PopPK of tadalafil in adult patients with PAH.

In both studies, patients were either not receiving PAHspecific therapy or were receiving ERAs, specifically bosentan or ambrisentan. Bosentan induces CYP3A4, which is the major pathway for tadalafil clearance, and concomitant bosentan treatment is known to reduce tadalafil exposure in patients. The LVIG study had insufficient information to evaluate the effect of the bosentan dose on tadalafil PK, and the PHIRST-1 study did not assess the effect of the bosentan 
dose. Ambrisentan does not affect tadalafil PK [10]; thus, patients taking ambrisentan were grouped with non-bosentan patients for analysis.

\subsection{Population Pharmacokinetic Modeling}

PopPK analyses were conducted using the nonlinear mixedeffects modeling program NONMEM ${ }^{\circledR}$ version 7.3.0 (ICON Development Solutions, Hanover, MD, USA). A pediatriconly model was first attempted and the paucity of samples in the absorption phase, attributable to the study design, resulted in difficulty estimating the absorption rate constant $(K a)$. Another limitation in this model attempt was demonstrated by inaccuracies in the estimations of $V / F$. Thus, the pediatric data from LVIG were pooled with the adult data from PHIRST-1 to better inform the model and facilitate parameter estimation. Samples with concentrations below the $0.5 \mathrm{ng} / \mathrm{mL}$ LLOQ and samples with inadequate sampling or dosing information were excluded from the analysis.

For PHIRST-1, a one-compartment structural model with first-order absorption and elimination provided the most appropriate description of tadalafil disposition (data on file). Likewise, for the pediatric model, a one-compartment model parameterized in terms of $C L / F, V / F, F$, and $K a$ was used to describe the tadalafil concentration-time data. The method of first-order conditional estimation with epsilon-eta interaction was used for all models [11]. Between-patient variability was assumed to be log-normally distributed and was tested on all PK parameters. Covariance between PK parameters (e.g. $C L / F$ and $V / F$ ) was assessed, as were different residual error models. Stepwise covariate modeling (SCM) was implemented using Perl-speaks-NONMEM [12] to identify significant covariates for determining forward inclusion (with significance defined as a drop of $\geq 6.635$ in the minimum value of the objective function [MOF], $p$ value of 0.01 with 1 degree of freedom) and backward elimination (which successively removes the least significant covariate whose removal does not increase the MOF by at least 10.828 [ $p$ value of 0.001 with 1 degree of freedom], until all remaining covariates are statistically significant).

The last backward model from the SCM process was further reduced in a stepwise manner, taking into account factors such as parameter precision, magnitude of effect of the covariate, and reduction in interindividual variability (a reduction of at least $5 \%$ was required to retain the covariate) to obtain the final pediatric PopPK model. Individual estimates of AUC were calculated as dose divided by empirical Bayesian estimates of $C L / F$. The average concentration over a 24-h dosing interval at steady state $\left(C_{\text {mean,ss }}\right)$ was calculated as the AUC over a dosing interval at steady state $\left(\mathrm{AUC}_{\mathrm{ss}}\right)$ divided by $24 \mathrm{~h}$. Half-life was calculated as 0.693 times the model-predicted $V / F$ divided by the model-predicted $C L / F$.

\subsection{Model Selection Criteria and Evaluation}

Selection between models was mainly based on the objective function value (OFV), the precision of parameter estimates, and visual predictive checks (VPCs). The minimum OFV provided by NONMEM that corresponds to approximately $-2 \times \log$ (likelihood) $[-2 L L]$ served as a criterion for model comparisons during model development. A decrease in - 2LL of 10.828 points for one additional parameter was regarded as a significant model improvement corresponding to a $p$ value of 0.001 for nested models. The Akaike information criterion (AIC), calculated as AIC $=-2 \mathrm{LL}+2 \times \mathrm{NP}$, where NP is the number of parameters in the model, was used for selection among non-nested models [13]. Precision of parameter estimates, expressed as the 2.5th and 97.5th percentiles of the parameter distribution, were computed from the analysis of 500 bootstrap data sets (sampling with replacement) using Perl-speaks-NONMEM [14]. Model parameter estimates were presented, together with the corresponding $95 \%$ CIs, as a measure of parameter imprecision computed from the results of the bootstrap analysis. The degree of intersubject variability was expressed as a coefficient of variation. A VPC with 1000 simulated studies was conducted for model diagnostics to ensure that the model maintained fidelity with the data used to develop it. Simulated and observed distributions were compared by calculating the median and the 10th and 90th percentiles for each. A prediction-corrected VPC [15] was conducted to investigate the agreement between the observed and predicted concentrations for both the adult and pediatric studies and to allow comparison of model performance across dosing regimens.

\section{Results}

The PK analyses included 1430 observations from 305 adult patients (PHIRST-1: 69 males and 236 females, 1102 observations) and 19 pediatric patients (LVIG: 6 males and 13 females, 328 observations) who received once-daily tadalafil at different dose levels (Table 1). LVIG had 19 predose samples below the LLOQ, all collected before the start of treatment, whereas PHIRST-1 had 17 samples below the LLOQ; thus, $2 \%$ of samples were excluded because they were below the LLOQ. PK samples that did not have adequate sampling or dosing information were also excluded from the analysis (LVIG: 5 samples; PHIRST-1: 35 samples).

Because the adult and pediatric data were combined for analysis, the effect of baseline body weight on PK parameter estimates was evaluated in the base model. Baseline body weight was deemed appropriate due to no notable withinpatient weight changes during the short time between the pediatric PK collection visits. Allometric principles were applied for testing body weight on clearance and volume 
Table 1 Summary of the two clinical trials included in the population PK analysis

\begin{tabular}{|c|c|c|c|c|}
\hline $\begin{array}{l}\text { Patient population (study) } \\
\text { [cohort, } n]\end{array}$ & $\begin{array}{l}\text { Age, years [median } \\
\text { (range)] }\end{array}$ & $\begin{array}{l}\text { Weight, kg [median } \\
\text { (range)] }\end{array}$ & $\begin{array}{l}\text { Daily treatment regimen/ } \\
\text { dose }\end{array}$ & PK sampling for tadalafil ${ }^{\mathrm{a}}$ \\
\hline \multicolumn{5}{|c|}{ Adult PAH patients (PHIRST-1) $[n=305]$} \\
\hline $\begin{array}{l}2.5 \mathrm{mg}(n=77) \\
10 \mathrm{mg}(n=77) \\
20 \mathrm{mg}(n=77) \\
40 \mathrm{mg}(n=74)\end{array}$ & $53.9(14.7-90.3)$ & $73.0(41.4-140)$ & $\begin{array}{l}\text { Placebo or tadalafil (tablet) } \\
2.5,10,20,40 \mathrm{mg}\end{array}$ & $\begin{array}{l}\text { Sparse sampling at weeks } \\
4,8,12 \text {, and } 16 \text {; week } 12 \\
\text { was specified as a trough } \\
\text { sample }\end{array}$ \\
\hline \multicolumn{5}{|l|}{ Pediatric PAH patients (LVIG) } \\
\hline $\begin{array}{l}\text { Heavy-weight cohort } \\
(\geq 40 \mathrm{~kg})[n=6]\end{array}$ & $14.6(11.3-17.6)$ & $49.0(43.0-76.0)$ & $\begin{array}{l}\text { Tadalafil (tablet) } \\
\text { Low: } 10 \mathrm{mg} \\
\text { High: } 20-40 \mathrm{mg}\end{array}$ & $\begin{array}{l}\text { Period } 1 \text { (10 weeks): } \\
\text { Serial PK sampling } \\
\text { Single dose: Day } 1 \text { (low }\end{array}$ \\
\hline $\begin{array}{l}\text { Middle-weight cohort } \\
\qquad(\geq 25 \text { to }<40 \mathrm{~kg})[n=7]\end{array}$ & $11.0(7.3-18.0)^{\mathrm{b}}$ & $30.1(26.1-37.3)$ & $\begin{array}{l}\text { Tadalafil (tablet) } \\
\text { Low: } 5 \mathrm{mg} \\
\text { High: } 10-20 \mathrm{mg}\end{array}$ & $\begin{array}{l}\text { dose) } \\
\text { Steady state: Day } 14 \text { (low } \\
\text { dose), Day } 49 \text { (high dose) }\end{array}$ \\
\hline $\begin{array}{l}\text { Light-weight cohort } \\
\quad(<25 \mathrm{~kg})[n=6]\end{array}$ & $5.0(2.5-8.0)$ & $14.7(10.0-23.9)$ & $\begin{array}{l}\text { Tadalafil }(2 \mathrm{mg} / \mathrm{mL} \text { sus- } \\
\text { pension }) \text { : } \\
\text { Low: } 2-4 \mathrm{mg} \\
\text { High: } 8-20 \mathrm{mg}\end{array}$ & $\begin{array}{l}\text { Times: predose and } 2,4,8, \\
\text { 12, and } 24 \text { h postdose } \\
\text { Period } 2 \text { ( } \geq 2 \text { years): } \\
\text { One sparse PK sample } 3 \\
\text { months after the start of } \\
\text { period } 2\end{array}$ \\
\hline
\end{tabular}

$n$ number of patients included in the PK dataset, $P A H$ pulmonary arterial hypertension, $P K$ pharmacokinetics

${ }^{a}$ Tadalafil concentrations were measured using a validated liquid-chromatography/tandem mass spectrometry method with a lower limit of quantification of $0.5 \mathrm{ng} / \mathrm{mL}$ (data on file)

${ }^{\mathrm{b}}$ One patient in the middle-weight cohort was aged 17 years, 11.5 months at the time of enrollment; due to programming standards, this patient's age was rounded to 18.0 for reporting

parameters [6, 7]; the effect was evaluated estimating the exponents and fixing them to the physiological values of 0.75 for $C L / F$ and 1 for $V / F$. In the model that estimated the exponents, the exponent for $V / F$ approximated to 1 . Thus, the base model was then tested with the exponent fixed to 1 and this resulted in no significant increase in the OFV. Unlike $V / F$, which approximated the theoretical exponent, the exponent for $C L / F$ approximated to 0 . The base model was then evaluated with the exponent on $C L / F$ fixed to 0 , thus removing the effect, which resulted in no significant increase in the OFV. Therefore, the best-fit base model had an effect of weight on $V / F$, fixed to the allometric scaling value of 1 , and had no effect of weight on $C L / F$. Intersubject variability was significant $(p<0.01)$ for $V / F$ and $C L / F$. A positive correlation between the intersubject variability associated with $V / F$ and intersubject variability associated with $C L / F$ was also significant and was retained in the base model. Upon establishing a base model, the effects of age, body weight, sex, dose, and concomitant bosentan treatment were assessed for clinical relevance, as appropriate, on $C L / F, V / F, F$, and $K a$.

Figure 1 displays the model-estimated pediatric PK parameters from LVIG and depicts the statistically significant covariate effects retained in the final model. The final PopPK model included effects of dose and age on $F$, an effect of bosentan on $C L / F$, and an effect of body weight on $V / F$. Specifically, bosentan increased $C L / F, V / F$ decreased with decreasing body weight, and $F$ decreased with increasing dose and decreasing age. The addition of dose and age covariates explained some of the variability on $F$, such that the interaction term between $C L / F$ and $V / F$ was no longer significant and was removed from the model.

The results of the final population model and the bootstrap analysis are shown in Table 2. The median and 95\% CI for each model parameter indicated that the parameters are well estimated.

The VPCs (Fig. 2a) showed that the model reliably predicted the observed concentrations and overall variability evident by the overlap of the individual observed concentrations with the distributions of simulated concentrations. Due to the small sample size of the pediatric study, the VPC used the 10th and 90th percentiles. Additional validation of the final model is evident from the goodness-of-fit plot, including the distribution of residual errors (Fig. 2b).

\section{Discussion}

The major route of tadalafil clearance is metabolism by CYP3A4, which has its lowest concentration and prevalence in the gastrointestinal tract and liver at birth and then increases with age $[16,17]$. It is possible that tadalafil is also metabolized by CYP3A7, which typically has similar substrate specificity as CYP3A4 although 
A

CL/F vs Bosentan Use

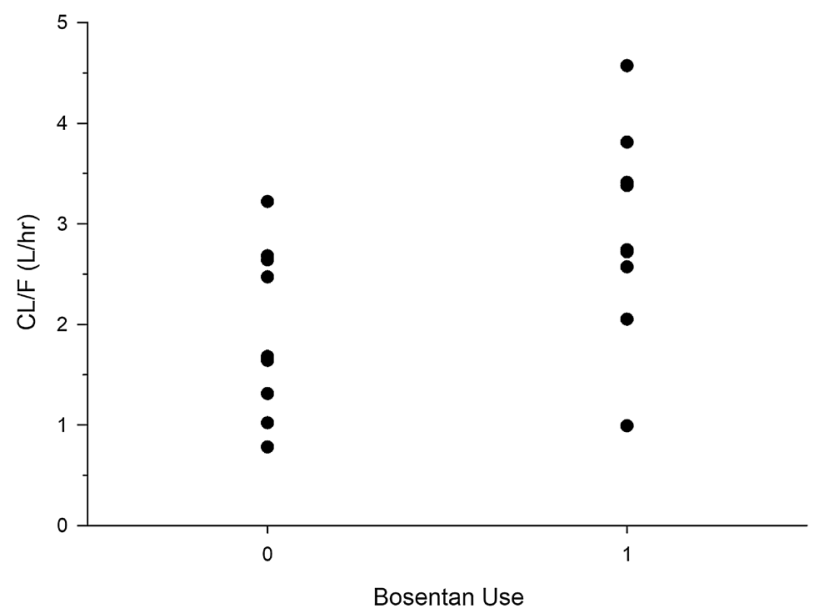

C

F vs Dose

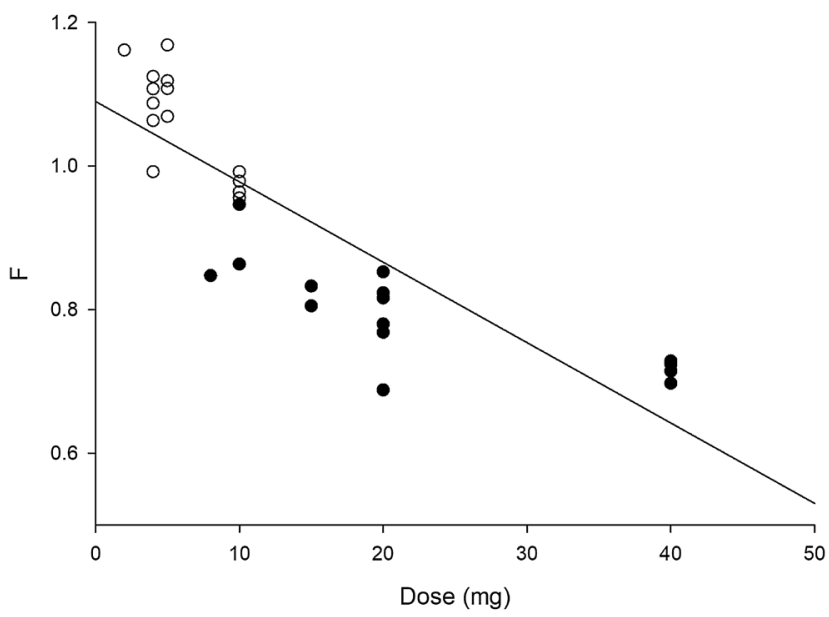

Fig. 1 Model-estimated pediatric PK parameters and relevant covariates from LVIG. Effect of a bosentan use on $C L / F$; $\mathbf{b}$ body weight on $V / F$; $\mathbf{c}$ dose on $F$; and $\mathbf{d}$ age on $F$. The effect of bosentan on $C L / F$ and the effect of body weight on $V / F$ were not affected by dose; high- and low-dose data overlap, therefore only the high dose is visible in plots

affinities can differ [18, 19]. CYP3A7 activity is the highest at birth and then diminishes until approximately 2 years of age, at which point its activity is very low [17]. Given the different directional changes in the activities of these enzymes from birth, and the potentially different affinities of the enzymes for tadalafil, the PopPK model cannot be extrapolated to ages younger than those assessed in the study. Since no patient $<2$ years of age was enrolled, no dosing recommendation was made for children in this age range.
B

V/F vs Weight

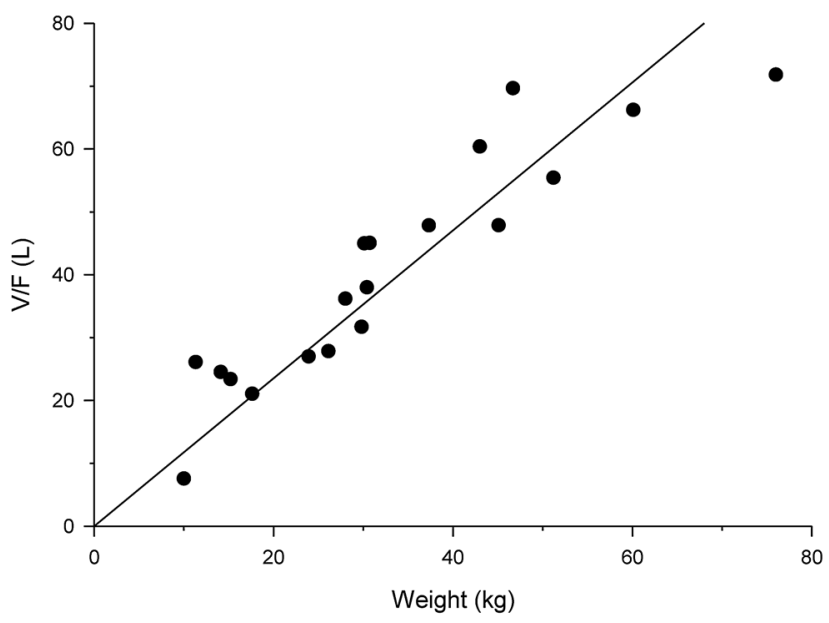

D

F vs Age

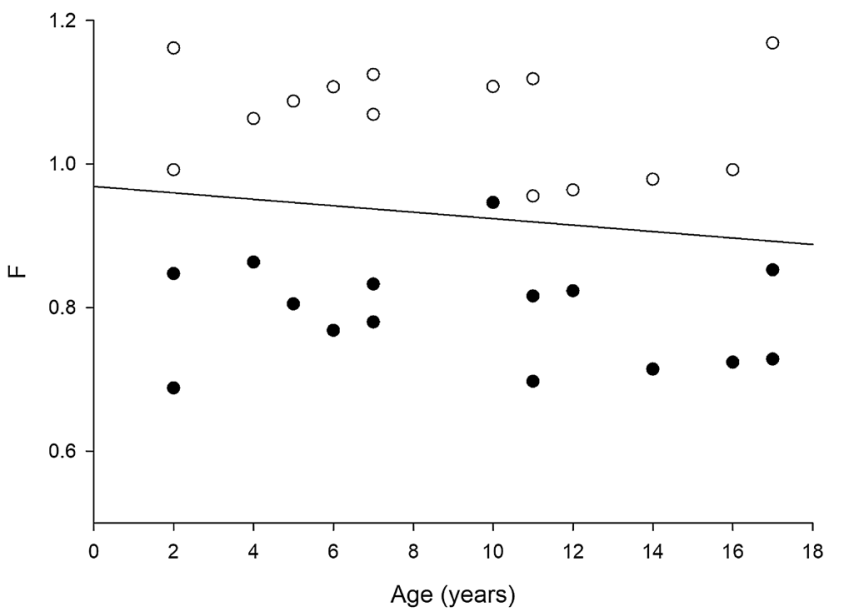

(a) and (b). Open and solid circles depict individual data points, and lines depict a linear regression through the points. $P K$ pharmacokinetic, $C L / F$ apparent clearance, $V / F$ apparent volume of distribution, $F$ bioavailability

Bosentan is a commonly coadministered ERA that induces CYP3A activity, thereby decreasing tadalafil exposure by up to $40 \%[9,20]$. About $50 \%$ of the adults (in PHIRST-1) and children (in LVIG) were coadministered bosentan (data on file), and the effect of bosentan on tadalafil $C L / F$ in LVIG was consistent with the known effect of bosentan on tadalafil $C L / F$ in adults (Table 3). Despite the known reduction in tadalafil exposure, tadalafil in adults is recommended at the same dose in patients taking bosentan and those not taking bosentan. In children, compensating 
Table 2 Pharmacokinetic and covariate parameters from the final population model

\begin{tabular}{|c|c|c|c|c|}
\hline Parameter description & $\begin{array}{l}\text { Population esti- } \\
\text { mate (\% SEE) }\end{array}$ & $\begin{array}{l}\text { Interpatient vari- } \\
\text { ability (\% SEE) }\end{array}$ & Estimated bootstrap ${ }^{\mathrm{a}}$ median $(95 \% \mathrm{CI})$ & $\begin{array}{l}\text { Interpatient vari- } \\
\text { ability bootstrap median } \\
(95 \% \mathrm{CI})\end{array}$ \\
\hline$K a\left(\mathrm{~h}^{-1}\right)$ & $0.860(10.9)$ & $201 \%^{\mathrm{b}}(26.4)$ & $0.891(0.652,1.22)$ & $198(91.5,390)$ \\
\hline \multicolumn{5}{|l|}{$C L / F(\mathrm{~L} / \mathrm{h})^{\mathrm{c}}$} \\
\hline Effect of non-bosentan & $-0.418(7.87)$ & & $-0.416(-0.474,-0.354)$ & \\
\hline Patients taking bosentan & $3.23(4.03)$ & $48.5 \%^{\mathrm{b}}(11.0)$ & $3.22(3.00,3.46)$ & $48.2(42.3,53.8)$ \\
\hline Not taking bosentan (calculated) & 1.88 & & & \\
\hline \multicolumn{5}{|l|}{$V / F[\mathrm{~L}]^{\mathrm{d}}$} \\
\hline $70 \mathrm{~kg}$ patient & $88.1(4.97)$ & $32.1 \%^{\mathrm{b}}(42.3)$ & $88.1(79.5,99.5)$ & $31.0(13.8,48.2)$ \\
\hline Effect of weight & 1 fixed & & & \\
\hline$F^{\mathrm{e}}$ & 1 fixed & & & \\
\hline Effect of dose (continuous) on $F$ & $-0.227(12.6)$ & & $-0.224(-0.280,-0.177)$ & \\
\hline Effect of age on $F$ & $0.100(45.6)$ & & $0.101(0.0121,0.190)$ & \\
\hline \multicolumn{5}{|l|}{ Residual error $^{\mathrm{f}}$} \\
\hline Additive (ng/mL) & $11.6(31.3)$ & & $43.3(17.4,70.0)$ & \\
\hline Proportional & $25.8 \%(10.4)$ & & $25.8(23.4,28.2)$ & \\
\hline
\end{tabular}

$C I$ confidence interval, $C L / F$ apparent clearance, $C V$ coefficient of variation, $F$ bioavailability, $K a$ absorption rate constant, $S E E$ standard error of the estimate reported using NONMEM, $V / F$ apparent volume of distribution

${ }^{a}$ The bootstrap method was a non-parametric bootstrap stratified by study

${ }^{\mathrm{b}}$ Reported as $\mathrm{CV}$, calculated using the equation $100 \% \cdot \sqrt{e^{\omega^{2}}-1}$, where $\omega^{2}$ is the NONMEM output for the intersubject variability of the parameter estimate

${ }^{\mathrm{c}} C L / F$ is modeled for a typical patient taking bosentan as $C L=(\mathrm{TVCL} \cdot \mathrm{BOS})+\mathrm{TVCL} \cdot(1+$ EffNoBos $) \cdot(1-\mathrm{BOS})$, where $C L$ is the parameter being modeled, TVCL is the typical value for the parameter, BOS is an indicator variable $(0=$ no, $1=$ yes $)$ for concomitant bosentan, and EffNoBos is the change in the parameter without bosentan

${ }^{\mathrm{d}} V / F$ is modeled for a typical patient as $V=\mathrm{TVV} \cdot \mathrm{COV}^{\mathrm{EffWt}}$, where $V$ is the parameter being modeled, TVV is the typical value for the parameter, COV is the ratio between the patient's body weight at study entry and a typical weight of an adult (70 kg), and EffWt is the effect of body weight on the parameter fixed to the allometric scaling value of 1

${ }^{\mathrm{e}} F$ is modeled for a typical patient as $F=\mathrm{TVF} \cdot\left(\frac{\mathrm{DOSE}}{16.27}\right)^{\mathrm{EffDoseF}} \cdot\left(\frac{\mathrm{AGEE}}{52.4}\right)^{\text {EffAgeF }}$, where $F$ is the parameter being modeled, TVF is the typical value for the parameter (in this case, fixed to 1), DOSE is the patient's reported dose, EffDoseF is the effect of dose on the parameter, AGEE is the patient's reported age at study entry, and EffAgeF is the effect of age on the parameter

${ }^{\mathrm{f}}$ Residual error is reported as (proportional) $\mathrm{CV}$, calculated using the equation $100 \% \cdot \sqrt{\sigma 1}$, and additive error reported as $\sqrt{x^{2} \cdot \sigma 1}$, where $x$ is the NONMEM output for the additive error term and $\sigma_{1}$ is the NONMEM output for the residual error term

for the reduced exposure caused by bosentan could require doses higher than that approved in adults. Therefore, like adults, tadalafil doses are recommended in pediatric patients regardless of concomitant bosentan, despite the reduced plasma tadalafil concentrations with the use of bosentan. At the recommended pediatric doses, plasma tadalafil concentrations, with or without concomitant bosentan, are expected to be comparable with those in adults with or without concomitant bosentan and within the range of AUCs produced by the 20-40 mg once-daily doses in adult PAH patients.

It should be noted that the effect of bosentan on tadalafil exposure could conceivably differ according to the bosentan dosing regimen. In the PHIRST-1 study, each adult maintained the same bosentan dosing regimen (all $\leq 125 \mathrm{mg}$ twice daily) throughout the study period, but no consistent regimen across adults was required. Similarly, in pediatric patients, bosentan dosing is prescribed based on age, weight, and physician decision, with doses ranging from $16 \mathrm{mg}$ twice daily for the lightest children (starting at $4 \mathrm{~kg}$ ), to a $125 \mathrm{mg}$ twice daily maintenance dose for children over the age of 12 years and with a body weight $>40 \mathrm{~kg}$ [21]. As described in the Adcirca ${ }^{\circledR}$ (tadalafil) prescribing information for adults, "In patients taking only Adcirca $40 \mathrm{mg}$ (i.e., without concomitant bosentan), the placebo-adjusted mean change in 6-MWD [min walk distance] was 44 meters. In adult patients taking Adcirca $40 \mathrm{mg}$ and concomitant bosentan therapy, the placebo-adjusted mean change in 6-MWD was 23 meters" [8]. Thus, both treatmentstadalafil alone and tadalafil with bosentan-show efficacy, but the efficacy appears lower with bosentan than without. Despite the lack of specific dosing information for bosentan, the current PopPK model was able to detect a significant categorical effect of bosentan on tadalafil $C L / F$. In this study, the differences between PK predictions in patients not taking or taking bosentan reflect the effect of bosentan on $C L / F$. 
Fig. 2 a Prediction-corrected VPC for the final population PK model stratified by the LVIG and PHIRST-1 studies (top row), and further stratified by weight category for LVIG and by dose for PHIRST-1 (bottom row). Black open circles represent individual observed concentrations. The solid black line depicts the median of observed data, while the orange shaded area represents the $95 \%$ confidence interval around the median of the simulated data. The dashed lines represent the 10th and 90th percentiles of observed concentrations, while the grey shaded areas represent simulated $95 \%$ confidence intervals. b Goodness-of-fit plots for the final population PK model. $P K$ pharmacokinetic, $V P C$ visual predictive check, $D D I$ daily dose in milligrams, $G R P$ pediatric weight category as follows: 1 , heavy-weight $\geq 40 \mathrm{~kg} ; 2$, middle-weight 25 to $<40 \mathrm{~kg}$; and 3, light-weight $<25 \mathrm{~kg}$. Tadalafil concentration units are $\mathrm{ng} / \mathrm{mL}$
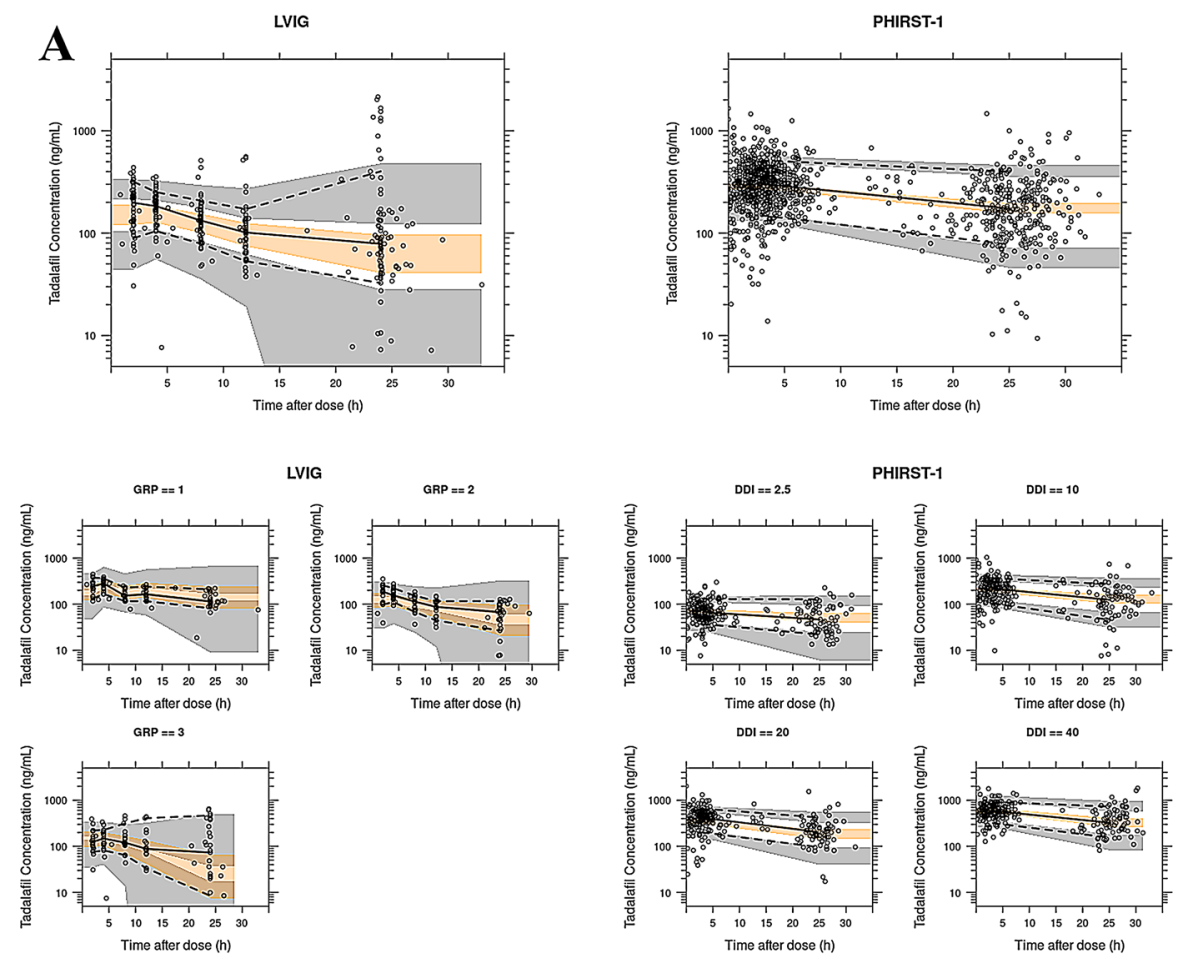

B
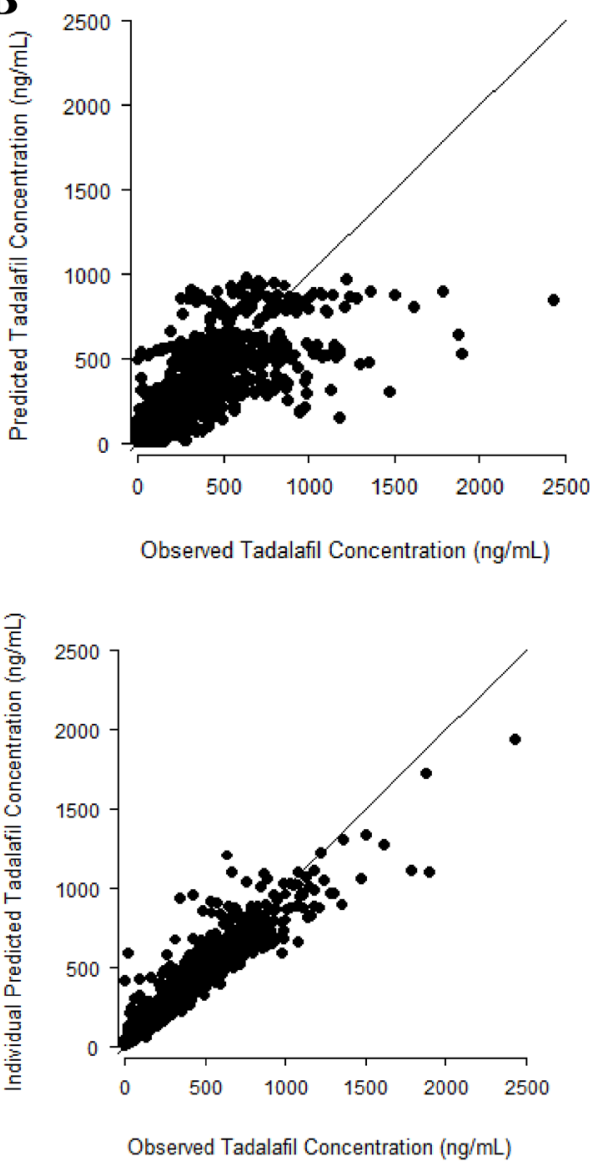
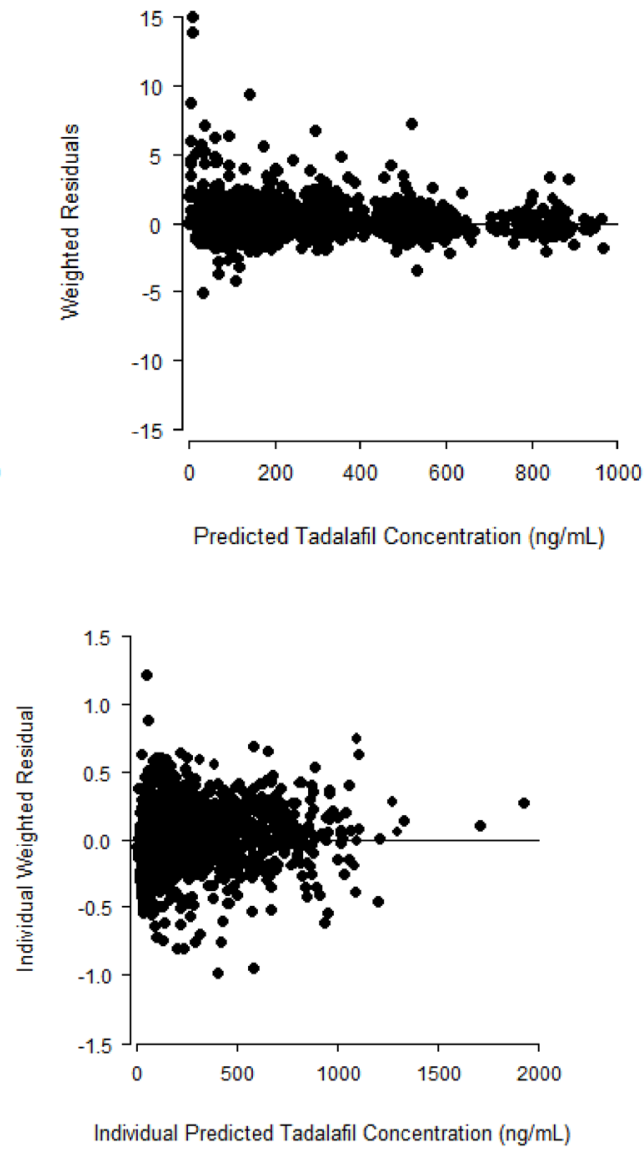


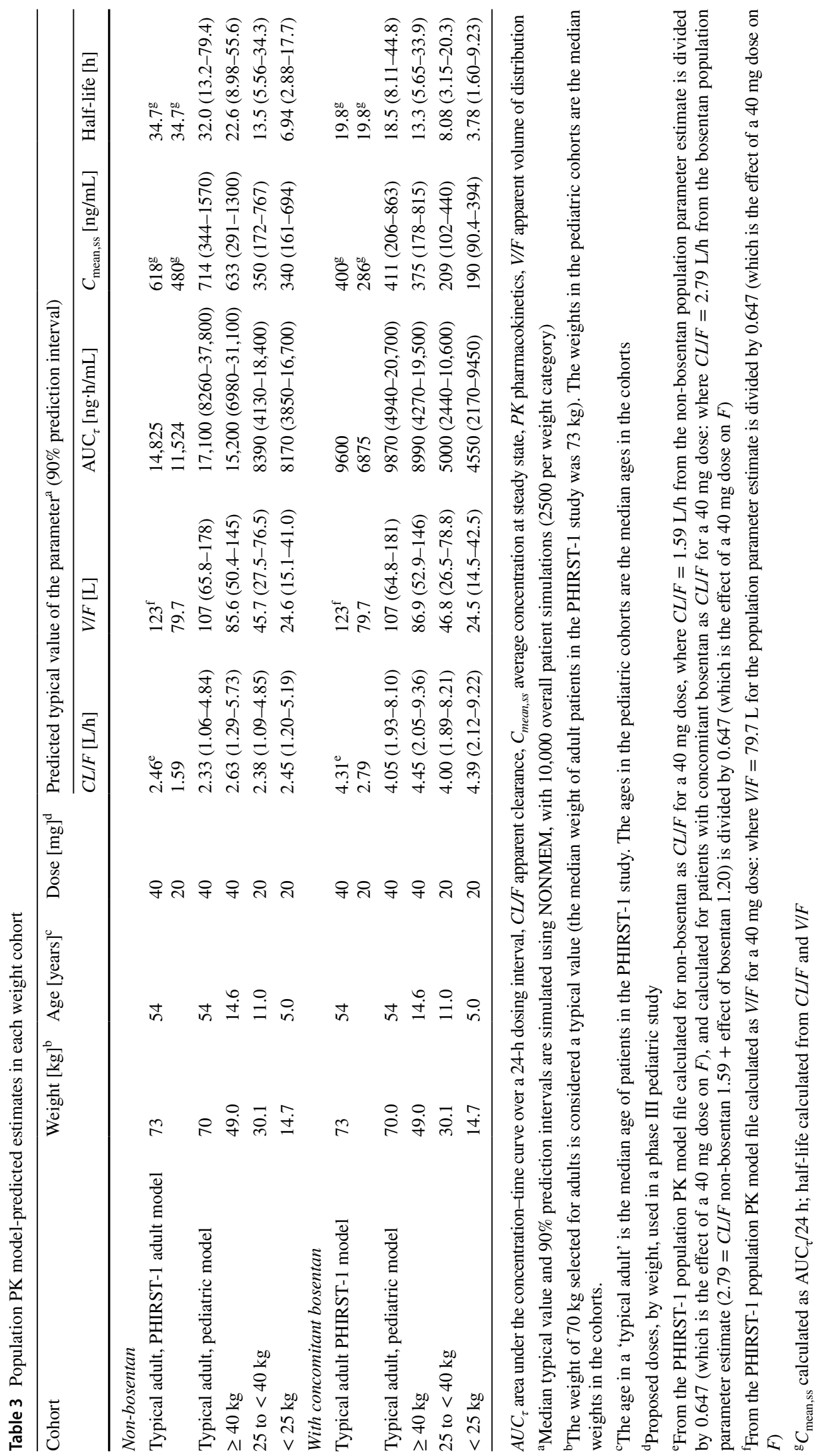


Based on allometric PK principles, we normally expect to see a difference in PK between adults and children in drug elimination, especially in clearance. In this case, as weight declined, the population estimate of tadalafil $V / F$ declined, while that of $C L / F$ did not change (Table 3 ). This led to shorter predicted half-lives as weight decreased, from $23 \mathrm{~h}$ in a typical $49 \mathrm{~kg}$ heavy-weight patient to $7 \mathrm{~h}$ in a typical $15 \mathrm{~kg}$ light-weight patient (Table 3 ). In patients taking bosentan, predicted half-lives decreased from $13 \mathrm{~h}$ in a typical $49 \mathrm{~kg}$ patient to $4 \mathrm{~h}$ in a typical $15 \mathrm{~kg}$ patient (Table 3 ). Consistent with the shorter half-lives, exposure in younger patients generally decreased. As was seen in noncompartmental analyses [5], PopPK simulations of the LVIG data showed that higher-than-expected doses were needed to reach the target AUC range in middle- and light-weight patients.

\subsection{Comparison of the Pediatric Model to the Adult Model}

One of the objectives of the LVIG study was to compare the pharmacokinetics of tadalafil in the pediatric population with historical data from adult patients with PAH. Several aspects of those comparisons warrant further discussion. While weight did not appear to be a significant covariate on $V / F$ in adult patients with $\mathrm{PAH}$, it is included in the combined model of adults and pediatric patients. The difference in the weight effect between models is likely due to the wider weight range in the combined analysis, which allowed elucidation of the weight effect on PK parameters. Body weights in the adult PHIRST-1 trial ranged from 41.4 to $140 \mathrm{~kg}$ (a 3.5-fold range), compared with 10 to $140 \mathrm{~kg}$ (a 14-fold range) in the combined adult and pediatric analysis.

In the LVIG pediatric model, the effect of dose on $\mathrm{F}$ is significant when dose is treated as a continuous variable across the $2.5-40 \mathrm{mg}$ range of doses in the study. This translates to $\mathrm{F}$ declining by $38 \%$ as the dose increases from 2.5 to $20 \mathrm{mg}$ within the same patient, and then declining by $15 \%$ as the dose increases from 20 to $40 \mathrm{mg}$. In comparison, in the adult model from PHIRST-1, F was not affected by increasing dose through $20 \mathrm{mg}$, but was 35\% lower for the $40 \mathrm{mg}$ dose than for the lower doses of $2.5,10$, and $20 \mathrm{mg}$ tested in the study.

Body weight and age are highly correlated in the pediatric population. Results of this analysis demonstrated that age significantly affected $F$ in the pediatric model. Dose also had a significant effect on $F$, but dose may also be confounded by different formulations administered to different weight groups. While formulation was tested and not found to be a significant covariate, the commercial tablet formulation was administered to the adults in PHIRST-1 and to the heavy- and middle-weight patients in LVIG, while lightweight patients in LVIG received tadalafil suspension. The light-weight patients were also the youngest patients and received the lowest doses, hence it could be hypothesized that the use of the suspension affected the estimate of $F$, as dose and age are confounded. A possible explanation is that in an adult bioequivalence study of $20 \mathrm{mg}$ tadalafil administered as a suspension or as a commercial tablet, the suspension was absorbed more slowly, with a $23 \%$ lower maximum observed tadalafil concentration $\left(C_{\max }\right)$, but just as extensively as the tablet with no difference in AUC (data on file). As a comparison between the adult and pediatric models, estimates of tadalafil PK parameters and exposures in a typical adult patient taking tadalafil $40 \mathrm{mg}$ once daily were predicted using the final pediatric PopPK model (Table 3).

\subsection{Dose Selection Based on Area Under the Concentration-Time Curve}

In line with observed data [5], modeling and simulation demonstrated that the doses studied in the pediatric population produced AUCs within range of the $90 \%$ prediction intervals of adult AUC at the approved adult dose of $40 \mathrm{mg}$ once daily (Table 3; Fig. 3).

Tadalafil PK parameter estimates (Table 3) were predicted for each pediatric weight cohort using the median weight and age in the respective cohort. Simulations were conducted in NONMEM, with 10,000 total patients (1250 for each of eight groups, including adults) to produce the 90\% prediction intervals around each parameter estimate. For comparison, the values predicted by the original adult PHIRST-1 model are also shown.

Dose selection in LVIG was premised on safety evaluations and on matching the tadalafil AUC in pediatric patients to a target range of AUC reported in adults. The pediatric PopPK model was used to predict tadalafil doses by weight cohort and bosentan use that, in pediatric patients, would produce the AUC estimated in adult PAH patients in PHIRST-1 taking $40 \mathrm{mg}$ once daily. The PopPK model predicts that to reach an adult-matched AUC of $9600 \mathrm{ng} \cdot \mathrm{h} / \mathrm{mL}$ in patients taking bosentan, pediatric patients should receive doses of 43-57 $\mathrm{mg}$ depending on weight, with lighter patients receiving higher doses than heavier patients. The higher dose in lighter patients reflects an effect of age on $F$, which decreases with decreasing age, and an effect of weight on $V / F$, which leads to shorter halflives in lower-weight patients. The same trend is apparent for patients not taking bosentan, although the target AUC and the specific model-predicted tadalafil doses differ.

The PopPK model predicts that doses of $\geq 38 \mathrm{mg}$ in pediatric patients would produce the AUC reported in adult patients taking $40 \mathrm{mg}$ once daily. During $40 \mathrm{mg}$ once-daily dosing across all pediatric age groups, the 


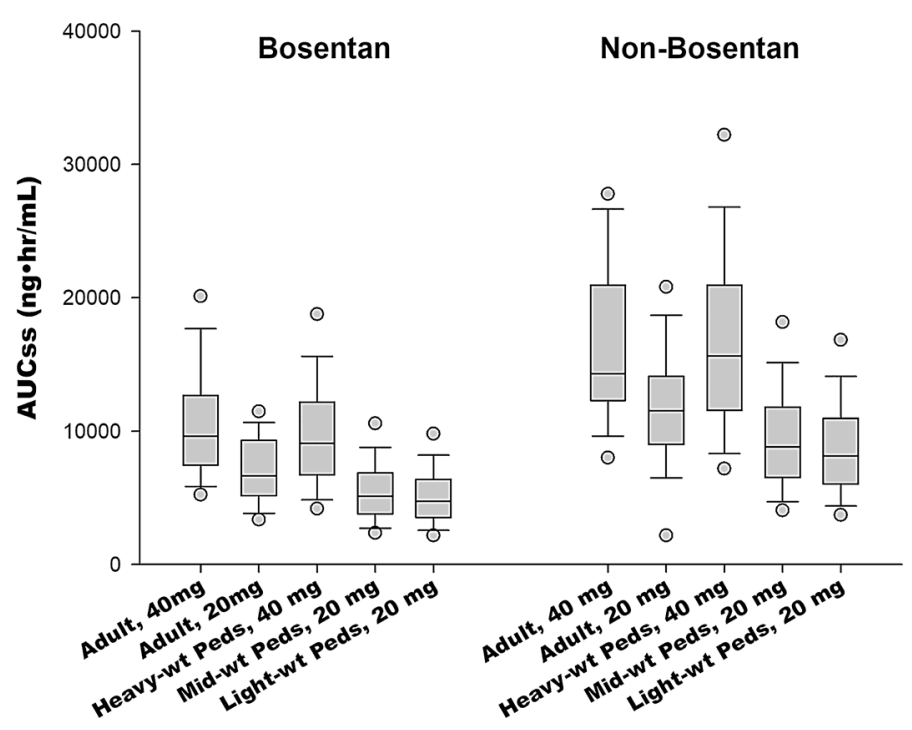

Fig. 3 The boundary of the box closest to zero indicates the 25th percentile, a line within the box marks the median, and the boundary of the box farthest from zero indicates the 75th percentile. Whiskers (error bars) above/below the box indicate the 90th/10th percentiles, and open circles represent the 5th and 95th percentiles. Simulated

median AUC in pediatric patients not taking bosentan approximates that in adults taking $40 \mathrm{mg}$ tadalafil without bosentan, and in pediatric patients taking bosentan, the median AUC is between the median AUCs in adults taking 20 or $40 \mathrm{mg}$ tadalafil with bosentan. In clinical practice, pediatric patients are unlikely to receive a dose above $40 \mathrm{mg}$ because that is the highest approved dose for $\mathrm{PAH}$ in adults.

\subsection{Considerations for Dose Selection}

In general, safety data in LVIG were generated at tadalafil AUCs within the targeted range and did not constrain dose selection during the study [5]. The safety of tadalafil treatment at doses above $20 \mathrm{mg}$ was not assessed in the two lighter-weight pediatric groups; however, the PopPK model was used to simulate the high dose of $20 \mathrm{mg}$ (middle- and light-weight) or $40 \mathrm{mg}$ (heavy-weight) in each cohort, as well as higher doses for consideration in the middle- and light-weight groups. Doses selected for further evaluation in a phase III pediatric study for children aged $\geq 2$ years are shown in Fig. 4a.

The approach to AUC matching a $40 \mathrm{mg}$ once-daily dose in adults in the pediatric study assumed that the shapes of the concentration-time profiles in pediatric patients would mimic those in adults, hence differences in AUC in patients aged $<18$ years would reflect proportional differences in concentrations at individual time adult data from PHIRST-1; simulated pediatric data from LVIG; heavy-weight patients weigh $>40 \mathrm{~kg}$, mid-weight patients weigh $25-40 \mathrm{~kg}$, and light-weight weigh $<25 \mathrm{~kg}$. AUC area under the curve, $A U C_{s s}$ steady-state area under the curve, Mid-wt mid weight, $Q D$ once daily, $w t$ weight

points during the profile. As shown in Fig. 4b, with or without bosentan, the PopPK model predicts that the PK profile of the heavy-weight group matches adults, but in middle- and light-weight patients, $C_{\max }$ is higher and halflife is shorter, leading to higher concentrations early in a 24-h profile, and lower concentrations later. At a dose of $20 \mathrm{mg}$ once daily, the $C_{\max }$ in the middle- and light-weight patients is not predicted to exceed that in adults or heavyweight patients (Fig. 4a), but at a $40 \mathrm{mg}$ once-daily dose it would (Fig. 4b). The effect of weight and age on $C_{\max }$ would appear to limit doses to $<40 \mathrm{mg}$ in the middleand light-weight groups, as $40 \mathrm{mg}$ doses were not tested and therefore the effect of higher $C_{\max }$ on safety was not evaluated.

Also pertinent to consideration of whether patients with a body weight $<40 \mathrm{~kg}$ should receive $20 \mathrm{mg}$ or should receive higher doses is the expected relative efficacy of 20 versus $40 \mathrm{mg}$. Following 16 weeks of tadalafil treatment in adult PAH patients, the model-predicted increase in 6-min walk distance is more than 30 meters for the 20 and $40 \mathrm{mg}$ doses, irrespective of bosentan use. Only a small difference of $<3$ meters in the modelpredicted 6 -min walk response was predicted between patients taking $20 \mathrm{mg}$ tadalafil and patients taking $40 \mathrm{mg}$ tadalafil [4] (Table 4).

Although the average AUCs in middle- and light-weight patients receiving $20 \mathrm{mg}$ are predicted to be lower than the average AUC in adults receiving $20-40 \mathrm{mg}$ once daily 
Fig. 4 Simulated tadalafil concentration-time profiles over a dosing interval at steady-state in the three pediatric weight cohorts and in adults with (left panel) or without (right panel) concomitant bosentan a at proposed phase III doses for children aged $\geq 2$ years; $\mathbf{b}$ with adult and all pediatric patients taking $40 \mathrm{mg}$ of tadalafil; or c light-weight cohort illustrating overlap to adult concentrations during 20 or $40 \mathrm{mg}$ once-daily dosing. Lines represent the predicted average concentrations at steady state based on 1250 simulations in each cohort. Pediatric cohorts were simulated using the PopPK model developed in LVIG, while the profile in adults was simulated using the PopPK model from PHIRST-1. 'Time' on the $x$-axis is time from the first dose, hence these profiles represent steady-state profiles after the eighth once-daily dose. Shaded regions represent the $90 \%$ prediction interval. LVGY is another name for the PHIRST-1 study. PopPK population pharmacokinetics, $Q D$ once daily
A

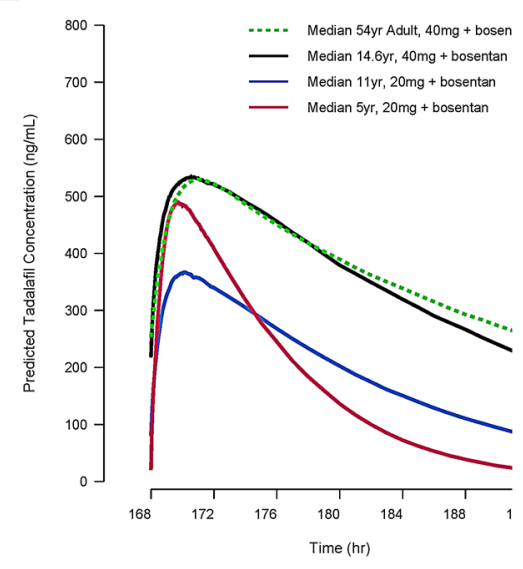

B
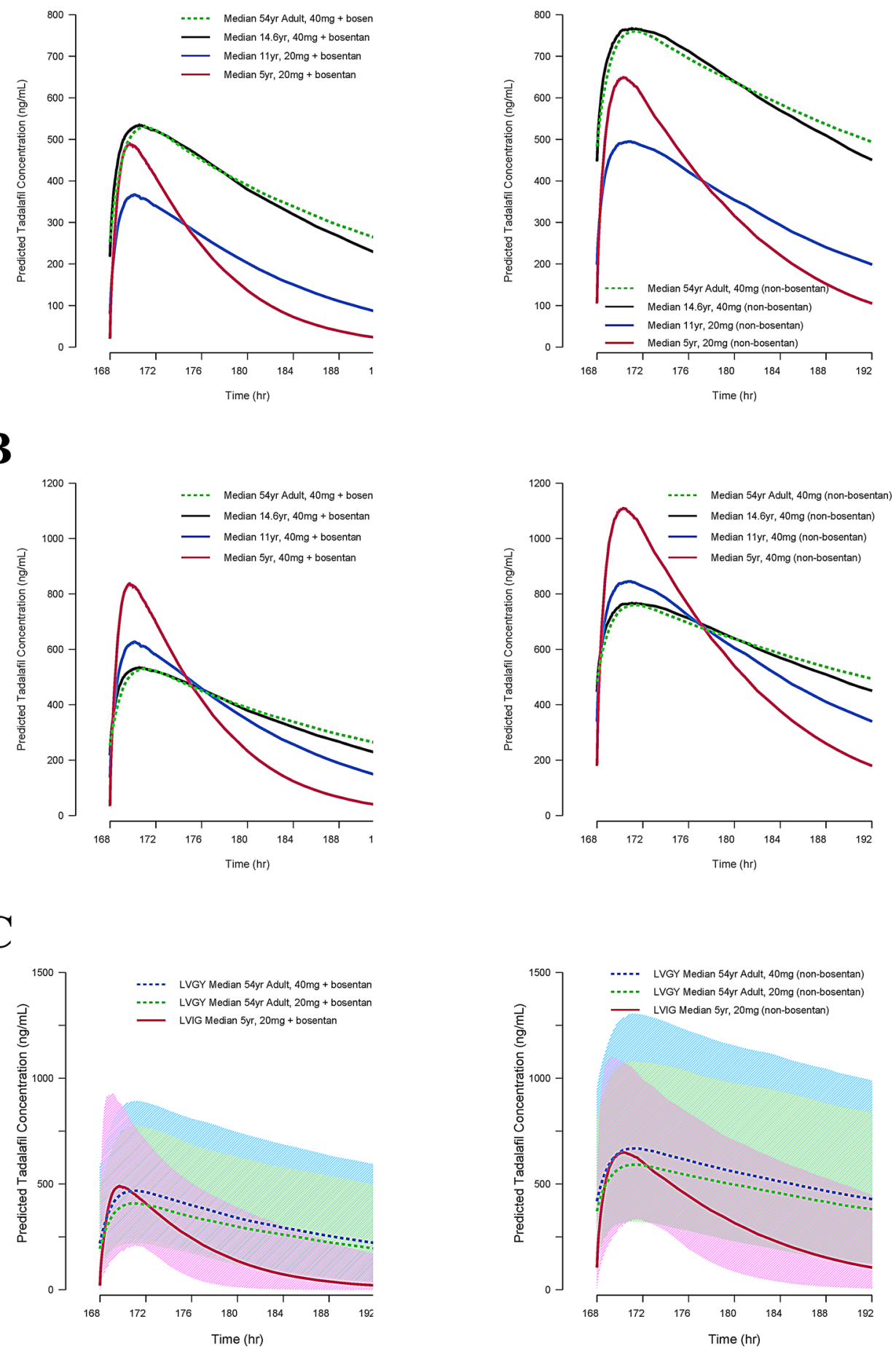

(Fig. 3), the simulated concentration-time profiles shown in Fig. 4c suggest that even in the light-weight cohort, concentrations within a dosing interval would largely overlap the adult concentrations during 20 or $40 \mathrm{mg}$ oncedaily dosing. Exposures in the current study were within the range of exposure in adult patients with PAH taking $20-40 \mathrm{mg}$ once daily.

\section{Conclusion}

A one-compartment PopPK model parameterized in terms of $F, K a, C L / F$, and $V / F$ described the data well. The final PopPK model of tadalafil in pediatric patients included an effect of weight on $V / F$ but not on $C L / F$. Other covariate effects were that bosentan increased $C L / F$ to a similar 
Table 4 Week 16 model-predicted 6-min walk response assuming the median, 10th, and 90th percentiles of $\mathrm{AUC}_{\mathrm{ss}}$ at each dose for adult patients in the PHIRST-1 study (adult model)

\begin{tabular}{llllll}
\hline Dose, $\mathrm{mg}$ & \multicolumn{2}{l}{ No bosentan } & & \multicolumn{2}{l}{ Concomitant bosentan } \\
\cline { 2 - 3 } & $\mathrm{AUC}_{\mathrm{ss}}(\mathrm{ng} \cdot \mathrm{h} / \mathrm{mL})^{\mathrm{a}}$ & Increase in 6-min walk $[\mathrm{m}]^{\mathrm{a}}$ & & $\mathrm{AUC}_{\mathrm{ss}}(\mathrm{ng} \cdot \mathrm{h} / \mathrm{mL})^{\mathrm{a}}$ & Increase in 6-min walk $[\mathrm{m}]^{\mathrm{a}}$ \\
\hline 2.5 & $1950(913-3740)$ & $25.1(21.2-29.0)$ & $33.4(27.4-37.0)$ & $2900(680-2130)$ & $22.0(19.8-25.6)$ \\
10 & $6940(2870-10,900)$ & $37.4(32.5-40.0)$ & $6875(4390-10,600)$ & $33.3(30.1-36.7)$ \\
40 & $11,500(6180-15,400)$ & $39.6(36.3-45.3)$ & $9600(5910-17,300)$ & $35.9(32.2-41.0)$ \\
\hline
\end{tabular}

Source: Data on file

$A U C_{s s}$ steady-state area under the concentration-time curve, $m$ meter(s)

${ }^{\text {a }}$ Median (10th - 90th percentile)

magnitude as that in adult PAH patients, V/F decreased with decreasing body weight, and $F$ decreased with increasing dose and decreasing age. The effect of sex was evaluated and was not significant.

Plasma tadalafil concentrations in pediatric patients aged 2 to $<18$ years were similar to those in adults at similar doses. However, the predicted $C_{\max }$ during $40 \mathrm{mg}$ once-daily dosing in middle- and light-weight patients was higher than the predicted $C_{\max }$ during $40-\mathrm{mg}$ once-daily dosing in adults, which constrained dose recommendations in these pediatric weight groups. The results from PopPK modeling and simulation support a dose of $40 \mathrm{mg}$ oncedaily in pediatric patients with a body weight $\geq 40 \mathrm{~kg}$, and a dose of $20 \mathrm{mg}$ once daily in patients with a body weight $<40 \mathrm{~kg}$ and aged $\geq 2$ years, for further evaluation in a phase III pediatric study.

Acknowledgements The authors would like to thank the investigators for the conduct of this study, and all the patients for their participation in the trials. Emmanuel Chigutsa, $\mathrm{PhD}$, Timothy Waterhouse, $\mathrm{PhD}$, and $\mathrm{Lan} \mathrm{Ni}, \mathrm{PhD}$, provided review and feedback during the population pharmacokinetic model development in the LVIG study. Kalyan Pulpika and Swati Bhandari, employees of Eli Lilly India Services Private Limited, provided writing support.

\section{Declarations}

Funding This study was funded by Eli Lilly and Company.

Conflict of interest Lisa Ferguson-Sells and Baohui Li are employees of Eli Lilly and Company and own stocks. David Small and Nieves Velez de Mendizabal are former employees of Eli Lilly and Company. NVM is an employee of Metrum Research Group, Tariffville, CT, USA, and DS is an employee of Karuna Therapeutics, Boston, MA, USA.

Ethics approval Both studies (LVIG and PHIRST-1) were conducted in accordance with consensus ethics principles derived from international ethics guidelines, including the Declaration of Helsinki and Council for International Organizations of Medical Sciences (CIOMS) International Ethical Guidelines; the International Council for Harmoniza- tion (ICH) Good Clinical Practice (GCP) Guideline [E6]; and applicable local laws and regulations. The local Ethics Review Committees approved the protocols, and written informed consent was obtained from patients or legal guardians prior to a patient's participation.

Consent to participate Informed consent was obtained from all individual participants included in the study.

Consent for publication Not applicable.

Availability of data and material Eli Lilly provides access to all individual participant data collected during the trial, after anonymization, with the exception of PK or genetic data. Data are available to request 6 months after the indication studied has been approved in the US and EU, and after primary publication acceptance, whichever is later. No expiration date of data requests is currently set once data are made available. Access is provided after a proposal has been approved by an independent review committee identified for this purpose and after receipt of a signed data sharing agreement. Data and documents, including the study protocol, statistical analysis plan, clinical study report, and blank or annotated case report forms, will be provided in a secure data sharing environment. For details on submitting a request, see the instructions provided at http://www.vivli.org.

Code availability Not applicable.

Author contributions LF-S, BL, and DS conceptualized and designed the study; LF-S and BL acquired data for the study; and LF-S, NVM, $\mathrm{BL}$, and DS analyzed and interpreted the data, drafted the paper, and critically revised the paper.

Open Access This article is licensed under a Creative Commons Attribution-NonCommercial 4.0 International License, which permits any non-commercial use, sharing, adaptation, distribution and reproduction in any medium or format, as long as you give appropriate credit to the original author(s) and the source, provide a link to the Creative Commons licence, and indicate if changes were made. The images or other third party material in this article are included in the article's Creative Commons licence, unless indicated otherwise in a credit line to the material. If material is not included in the article's Creative Commons licence and your intended use is not permitted by statutory regulation or exceeds the permitted use, you will need to obtain permission directly from the copyright holder. To view a copy of this licence, visit http://creativecommons.org/licenses/by-nc/4.0/. 


\section{References}

1. Rich S, Rubin LJ, Abenhail L, et al. Executive summary from the world symposium on primary pulmonary hypertension (Evian, France; September 6-10, 1998). Geneva: World Health Organization; 1998. p. 1-27.

2. Barst RJ, McGoon M, Torbicki A, et al. Diagnosis and differential assessment of pulmonary arterial hypertension. J Am Coll Cardiol. 2004;43(12 Suppl S):40S-47S.

3. Beghetti M. Paediatric pulmonary hypertension: monitoring progress and identifying unmet needs. Eur Respir Rev. 2009;18(111):18-23.

4. Galiè N, Brundage BH, Ghofrani HA, et al. Tadalafil therapy for pulmonary arterial hypertension. Circulation. 2009;119(22):2894-903.

5. Small D, Ferguson-Sells L, Dahdah N, Bonnet D, Landry J, Li B. Pharmacokinetics and safety of tadalafil in a paediatric population with pulmonary arterial hypertension: a multiple ascending-dose study. Br J Clin Pharmacol. 2019;85(10):2302-9.

6. Holford NH. A size standard for pharmacokinetics. Clin Pharmacokinet. 1996;30(5):329-32.

7. Anderson BJ, Holford NH. Mechanistic basis of using body size and maturation to predict clearance in humans. Drug Metab Pharmacokinet. 2009;24(1):25-36.

8. Adcirca [prescribing information]. Indianapolis: Eli Lilly and Company; 2009. Available at: https://www.accessdata.fda.gov/ drugsatfda_docs/label/2009/022332lbl.pdf.

9. Wrishko RE, Dingemanse J, Yu A, Darstein C, Phillips DL, Mitchell MI. Pharmacokinetic interaction between tadalafil and bosen$\tan$ in healthy male subjects. J Clin Pharmacol. 2008;48(5):610-8.

10. Spence R, Mandagere A, Harrison B, Dufton C, Boinpally R. No clinically relevant pharmacokinetic and safety interactions of ambrisentan in combination with tadalafil in healthy volunteers. J Pharm Sci. 2009;98(12):4962-74.

11. Beal S, Sheiner LB, Boeckmann A, Bauer RJ, editors. NONMEM 7.3.0 users guides $(1989,2013)$. Ellicott City: Icon Development Solutions; 2013.
12. Lindbom L, Pihlgren P, Jonsson EN. PsN-Toolkit-a collection of computer intensive statistical methods for non-linear mixed effect modeling using NONMEM. Comput Methods Progr Biomed. 2005;79(3):241-57.

13. Ludden TM, Beal SL, Sheiner LB. Comparison of the Akaike Information Criterion, the Schwarz criterion and the $F$ test as guides to model selection. J Pharmacokinet Biopharm. 1994;22(5):431-45.

14. Lindbom L, Ribbing J, Jonsson EN. Perl-speaks-NONMEM (PsN) - a Perl module for NONMEM related programming. Comput Methods Progr Biomed. 2004;75(2):85-94.

15. Bergstrand M, Hooker AC, Wallin JE, Karlsson MO. Prediction-corrected visual predictive checks for diagnosing nonlinear mixed-effects models. AAPS J. 2011;13(2):143-51.

16. Lacroix D, Sonnier M, Moncion A, Cheron G, Cresteil T. Expression of CYP3A in the human liver-evidence that the shift between CYP3A7 and CYP3A4 occurs immediately after birth. Eur J Biochem. 1997;247(2):625-34.

17. Stevens J, Hines R, Gu C, et al. Developmental expression of the major human hepatic CYP3A enzymes. J Pharmacol Exp Ther. 2003;307:573-82.

18. Williams JA, Ring BJ, Cantrell VE, et al. Comparative metabolic capabilities of CYP3A4, CYP3A5, and CYP3A7. Drug Metab Dispos. 2002;30(8):883-91.

19. Takahiro R, Nakamura S, Kohno H, et al. Contribution of CYP3A isoforms to dealkylation of PDE5 inhibitors: a comparison between sildenafil $N$-demethylation and tadalafil demethylenation. Biol Pharm Bull. 2015;38(1):58-65.

20. Parikh V, Bhardwaj A, Nair A. Pharmacotherapy for pulmonary arterial hypertension. J Thorac Dis. 2019;11(12):S1767-81.

21. Tracleer [prescribing information]. South San Francisco: Actelion Pharmaceuticals US, Inc; 2009. Available at: https://www.acces sdata.fda.gov/drugsatfda_docs/label/2017/209279s000lbl.pdf.

\section{Authors and Affiliations}

\section{Lisa Ferguson-Sells ${ }^{1}\left[\right.$ (D) Nieves Velez de Mendizabal ${ }^{1} \cdot$ Baohui $^{L^{1}}{ }^{1} \cdot$ David Small $^{1}$}

\section{Lisa Ferguson-Sells}

lis@lilly.com

1 Eli Lilly and Company, Indianapolis, IN 46285, USA 